\title{
COMMUTATIVE EXTENSION OF PARTIAL AUTOMORPHISMS OF GROUPS
}

\section{Introduction.}

\author{
by C. G. CHEHATA \\ (Received 5th June, 1953)
}

Let $\mu$ be an isomorphism which maps a subgroup $A$ of the group $G$ onto a second subgroup $B$ (not necessarily distinct from $A$ ) of $G$; then $\mu$ is called a partial automorphism of $G$. If $A$ coincides with $G$, that is if the isomorphism is defined on the whole of $G$, we speak of a total automorphism; this is what is usually called an automorphism of $G$. A partial (or total) automorphism $\mu^{*}$ extends or continues a partial automorphism $\mu$ if $\mu^{*}$ is defined for, at least, all those elements for which $\mu$ is defined, and moreover $\mu^{*}$ coincides with $\mu$ where $\mu$ is defined.

It is known (2) that any partial automorphism of a group can always be extended to a total automorphism of a supergroup, and even to an inner automorphism of a supergroup. Moreover, any number of partial automorphisms can be simultaneously extended to inner automorphisms of one and the same supergroup. In this paper conditions are investigated which ensure that two partial automorphisms can be extended to commutative (or permutable) automorphisms of a supergroup.

Sufficient conditions are derived in $\S 2$, conditions which are too restrictive to be necessary as well, but which are sufficiently wide to give the following special case as a corollary :

If $\mu$ maps $A \subseteq G$ isomorphically onto $B \subseteq G$ and if $\nu$ maps $C \subseteq G$ isomorphically onto $D \subseteq G$, and if

$$
A \cap C=B \cap C=A \cap D=B \cap D=\{1\} \text {, }
$$

then $\mu$ and $\nu$ can be extended to commutative automorphisms $\mu^{*}$ and $\nu^{*}$ of a supergroup $G^{*}$ of $G$.

The principal tool throughout is the free product of two groups with one amalgamated subgroup.

I am indebted to Dr. B. H. Neumann for his advice and continuous help during the work.

§1. Definitions and Lemmas.

In this paragraph we explain what is meant by an incomplete group, group amalgams, homomorphism of an amalgam, canonic group, canonic homomorphism and generalized free products. $\dagger$ We then state Lemma 1 which is proved by Hanna Neumann (3), and prove some other lemmas which will be required later on.

\section{Definition 1.}

An incomplete group is a set of elements with a group operation defined for some pairs of its elements.

Then we call $A$ an amalgam of groups $G_{i}$ if $A$ is an incomplete group consisting of the elements of the groups $G_{i}$ with the product of two elements of $A$ defined if, and only if, they both lie in (at least) one and the same group $G_{i}$. We call $G_{i}$ the constituent groups of the amalgam and denote by $H_{i j}=H_{j i}(i \neq j)$ the intersection of $G_{i}$ and $G_{j} ; H_{i j}$ may contain the unit element alone.

$†$ These definitions are adopted from a paper under publication by B. H. Neumann and H. Neumann for the purpose of making this paper self-contained. 
Definition 2.

A homomorphism of an amalgam $A$ into a group $P$ is a mapping $\phi$ of $A$ into $P$ such that if $a$ and $b$ are two elements of $A$ whose product is defined in $A$, then

in $P$.

$$
(a b) \phi=a \phi b \phi
$$

Definition 3.

We associate with the amalgam $A$ the group $P^{*}$ which is generated by elements

$$
a^{*}=a \phi^{*}
$$

corresponding to the elements $a$ of $A$; with the defining relations

$$
a^{*} b^{*}=c^{*}
$$

whenever $a^{*}=a \phi^{*}, b^{*}=b \phi^{*}, c^{*}=c \phi^{*}$ and

$$
a b=c
$$

in $A$. We call $P^{*}$ the canonic group of $A$ and $\phi^{*}$ the canonic homomorphism of $A$.

If the mapping $\phi^{*}$ is one-to-one, then we say that $P^{*}$ is the free product of the $G_{i}$ with amalgamated subgroups $H_{i j}$.

Hanna Neumann proved the following lemma (3, Corollary 8.11):

Lemma 1.

Let $P$ be the free product of two groups $G_{1}$ and $G_{2}$ with an amalgamated subgroup $H$; we use the notation

$$
P=\left\{G_{1} * G_{2} ; H\right\}
$$

Then if $A_{1}$ and $A_{2}$ are subgroups of $G_{1}$ and $G_{2}$ respectively which have the same intersection $B$ with $H$, then they generate in $P$ the free product of $A_{1}$ and $A_{2}$ with the amalgamated subgroup $B$.

\section{Lemma 2.}

Let

$$
P=\left\{G_{1} * G_{2} ; H\right\}
$$

and let $A_{1}$ and $A_{2}$ be subgroups of $G_{1}$ and $G_{2}$ respectively with $A_{1} \cap H=A_{2} \cap H=B$ as in Lemma 1. Let

Then

$$
\begin{aligned}
\left\{A_{1}, A_{2}\right\} & =Q . \\
Q \cap G_{i} & =A_{i} ; \quad i=1,2 .
\end{aligned}
$$

Proof :

By Lemma 1 we have

$$
Q=\left\{A_{1} * A_{2} ; B\right\}
$$

Choose $T_{i}$ as a set of left-hand coset representatives of $A_{i} \bmod B(i=1,2)$. Then $a \in A_{i}$ is uniquely expressed in the form

$$
a=t b ; \quad t \in T_{i}, \quad b \in B .
$$

Assume that $l \in T_{i}$, that is to say, $B$ is to be represented by the unit element. Then for any $q \in Q$ there exists a unique normal form

$$
q=t_{1} t_{2} \ldots t_{n} b,
$$

where each $t_{i} \in T_{1}-\{1\}$ or $T_{2}-\{1\}, b \in B$ and if $t_{i} \in T_{\alpha}$ then $T_{i+1} \bar{\epsilon} T_{\alpha}$. We call $n$ the length of $q$; thus $n=0$ for elements of $B$ and $n=1$ for elements of $A_{i}-B$. 
Choose $S_{i}$ as a set of left-hand coset representatives of $G_{i} \bmod H$ such that $S_{i} \supseteq T_{i}$. This is possible because if $t_{m} H=t_{n} H\left(m \neq n ; t_{m}, t_{n} \in T_{1}\right.$ say), then

$$
t_{m}^{-1} t_{n} \in H \text {, }
$$

but

$$
t_{m}^{-1} t_{n} \in A_{1} \text {, }
$$

thus

$$
t_{m}^{-1} t_{n} \in A_{1} \cap H=B
$$

contrary to the assumption that $T_{i}$ is a set of left-hand coset representatives of $A_{i} \bmod B$.

A normal form thus exists in $P$ with respect to $S_{i}$.

Let

$$
g \in Q \cap G_{i} \text {. }
$$

$g$ as an element in $Q$ has a normal form

$$
g=t_{1} t_{2} \ldots t_{n} b
$$

but as an element in $G_{i}$, it can be written uniquely as

$$
g=s_{i} h, \quad s_{i} \in S_{i}, \quad h \epsilon H .
$$

From the uniqueness of the representation we get

$$
n=1, \quad t_{1}=s_{i}, \quad b=h .
$$

Thus $g=t_{1} b \in A_{i}$, that is to say,

but since we obviously have

$$
Q \cap G_{i}=A_{i}
$$

$$
\begin{array}{ll} 
& Q \cap G_{i} \supseteq A_{i}, \\
\text { then } & Q \cap G_{i}=A_{i} .
\end{array}
$$

This completes the proof.

We mention without proof the following known result (1) :

Lemma 3.

Let $\mu$ be a homomorphism of the amalgam $A$ into a group $Q$. Then there is a homomorphism $\nu$ of the canonic group $P^{*}$ of $A$ into $Q$ such that

$$
\mu=\phi^{*} \nu
$$

where $\phi^{*}$ is the canonic mapping of $A$.

As an immediate consequence of Lemma 3 we have:

\section{Corollary :}

Let $G_{1}$ and $G_{2}$ be two groups with $U=G_{1} \cap G_{2}$, and let $\mu_{i}$ map $G_{i}$ homomorphically onto $H_{i}(i=1,2)$. Suppose that $U \mu_{1} \cong U \mu_{2}=V$ and that, more precisely, $u \mu_{1}=u \mu_{2}$ for all $u \epsilon U$. Then there exists a homomorphic mapping $\nu$ of

$$
P_{1}=\left\{G_{1} * G_{2} ; U\right\}
$$

onto any group $P_{2}$ generated by $H_{1}$ and $H_{2}$ such that $H_{1} \cap H_{2} \supseteq V$ where $\nu$ extends $\mu_{1}$ and $\mu_{2}$ simultaneously.

Proof.

For we can take $A$ as the amalgam whose constituent groups are $G_{1}$ and $G_{2} . P^{*}$ and $Q$ become $P_{1}$ and $P_{2}$ respectively, and the result follows immediately from Lemma 3. 
Lemma 4 .

Let $G_{1}$ and $G_{2}$ be two groups with $U=G_{1} \cap G_{2}$ and let $\mu_{i}$ map $G_{i}$ isomorphically onto $H_{i}(i=1,2)$. Suppose that $U \mu_{1} \cong U \mu_{2}=V$ and that, more precisely, $u \mu_{1}=u \mu_{2}$ for all $u \in U$. Then there exists an isomorphic mapping of

onto

$$
\begin{aligned}
& P_{1}=\left\{G_{1} * G_{2} ; U\right\} \\
& P_{2}=\left\{H_{1} * H_{2} ; V\right\},
\end{aligned}
$$

which extends $\mu_{1}$ and $\mu_{2}$ simultaneously.

Proof.

Since $U \mu_{1} \simeq U \mu_{2}=V$, then by the previous corollary there exists a homomorphic mapping $\nu$ of $P_{1}$ onto $P_{2}$ which extends both $\mu_{1}$ and $\mu_{2}$, that is to say

$$
\begin{aligned}
& P_{1} \nu=P_{2} \\
& \left.G_{i} \nu=G_{i} \mu_{i}=H_{i} ; \quad i=1,2 .\right\} .
\end{aligned}
$$

Since $\mu_{i}$ is an isomorphism, then its inverse $\mu_{i}^{-1}$ is an isomorphic mapping of $H_{i}$ onto $G_{i}$.

Again since $V \mu_{1}^{-1} \cong V \mu_{2}^{-1}=U$, then by the previous corollary there exists a homomorphic mapping $\nu^{\prime}$ of $P_{2}$ onto $P_{1}$ which extends $\mu_{1}^{-1}$ and $\mu_{2}^{-1}$ respectively, that is to say

$$
\left.\begin{array}{l}
P_{2} v^{\prime}=P_{1}, \\
H_{i} v^{\prime}=H_{i} \mu_{i}^{-1}=G_{i} ; \quad i=1,2 .
\end{array}\right\}
$$

Applying $\nu^{\prime}$ to (1.2) we get

$$
\begin{aligned}
& P_{1} \nu \nu^{\prime}=P_{2} \nu^{\prime}=P_{1}, \\
& G_{i} \nu \nu^{\prime}=H_{i} \nu^{\prime}=G_{i} \epsilon_{i},
\end{aligned}
$$

where $\epsilon_{i}$ is the identity mapping.

Applying $\nu$ to (1.3) we get

$$
\begin{aligned}
& P_{2} \nu^{\prime} \nu=P_{1} \nu=P_{2}, \\
& H_{i} \nu^{\prime} \nu=G_{i} \nu=H_{i} \epsilon_{i} .
\end{aligned}
$$

Thus $\nu v^{\prime}$ maps $P_{1}$ onto itself and on the constituent groups of $P_{1}$ it is equal to the identity mapping. Also $\nu^{\prime} \nu$ maps $P_{2}$ onto itself and on the constituent groups of $P_{2}$ it is equal to the identity mapping.

Hence $v$ and $\nu^{\prime}$ are reciprocal isomorphisms and the lemma follows.

\section{§. Sufficient Conditions.}

Denote by $A, B, C, D$ subgroups of a given group $G$ and assume that $\mu$ maps $A$ isomorphically onto $B$ and $\nu$ maps $C$ isomorphically onto $D$. Throughout this paragraph : $A, B, C, D, G, \mu$ and $\nu$ will always retain the same meaning.

For the existence of a group $G^{*} \supseteq G$ and two total automorphisms $\mu^{*}$ and $\nu^{*}$ of $G^{*}$ such that $\mu^{*} \nu^{*}=v^{*} \mu^{*}$ and $\mu^{*}, \nu^{*}$ extend $\mu, \nu$ respectively one condition is obviously necessary, namely the following :

$$
g \mu \nu=g \nu \mu
$$

whenever $g \mu, g_{\nu},(g \mu) \nu,(g \nu) \mu$ are defined, in other words whenever $g \epsilon A \cap C, g \mu \epsilon C$ and $g \nu \epsilon A$. Because if $G^{*}, \mu^{*}$ and $\nu^{*}$ exist, then for such an element $g$ we get :

and since $\mu^{*}$ commutes with $\nu^{*}$, then

$$
\begin{aligned}
& g \mu \nu=g \mu^{*} \nu^{*}, \\
& g \nu \mu=g \nu^{*} \mu^{*},
\end{aligned}
$$

$$
g \mu \nu=g_{\nu \mu} .
$$


We now show the sufficiency of certain conditions by proving the following theorem : Theorem 1.

For the existence of a supergroup $G^{*} \supseteq G$ with two commutative inner automorphisms $s$ and $t$ extending $\mu$ and $\nu$ respectively, it is sufficient that the following hold :

$$
\begin{aligned}
(A \cap C) \mu & =B \cap C, \\
(A \cap D) \mu & =B \cap D, \\
(A \cap C) \nu & =A \cap D, \\
(B \cap C) \nu & =B \cap D, \\
g \mu \nu & =g \nu \mu, \ldots
\end{aligned}
$$

whenever $g_{\mu}, g_{\nu},(g \mu) \nu$ and $\left(g_{\nu}\right) \mu$ are defined.

Proof.

The proof of this theorem is somewhat long and it will be effected by a number of lemmas. The main steps in the procedure are these: we take a sequence $\ldots, G_{-1}, G_{0}, G_{1}, \ldots$ of copies of the group $G$, that is to say a sequence of groups isomorphic to $G$, and construct certain free products $P_{i j}$ defined inductively for any $i<j$ and for $G_{i}, G_{i+1}, \ldots, G_{j}$ with certain amalgamated subgroups. We then prove that in $P_{i j}$ there exist two subgroups $Q_{i j}$ and $R_{i j}$ which are isomorphic under a mapping $\nu_{i j}$ that extends $\nu$. Lastly we form the union $U_{n=1}^{\infty} P_{-n,+n}$ and prove that it contains two subgroups which are isomorphic under a mapping $\nu$ that extends $\nu$, and that it possesses an automorphism $\mu^{*}$ which extends $\mu$ and commutes with $\bar{\nu}$. The proof will then be completed by applying a theorem due to G. Higman, B. H. Neumann and H. Neumann (2).

Now take a sequence of groups

$$
\ldots, G_{-1}, G_{0}, G_{1}, \ldots,
$$

each of which is isomorphic to $G$. Let under a fixed isomorphic mapping $\gamma_{i}$,

$$
G \gamma_{i}=G_{i} ; \quad i=0, \pm 1, \ldots .
$$

Each group $G_{i}$ contains subgroups $A_{i}, B_{i}, C_{i}, D_{i}$, which are the images of $A, B, C, D$ under the mapping $\gamma_{i}$ and possesses two isomorphisms $\mu_{i}$ and $\nu_{i}$ mapping $A_{i}$ onto $B_{i}$ and $C_{i}$ onto $D_{i}$ respectively, such that if

$$
\begin{array}{ll}
a_{i}=a \gamma_{i}, & a \in A, \\
b_{i}=b \gamma_{i}, & b \in B,
\end{array}
$$

then $a_{i} \mu_{i}=b_{i}$ if $a_{\mu}=b$. In other words,

Similarly one defines $\nu_{i}$ to be

$$
\begin{aligned}
\mu_{i} & =\gamma_{i}^{-1} \mu \gamma_{i} . \\
\nu_{i} & =\gamma_{i}^{-1} \nu \gamma_{i} .
\end{aligned}
$$

If we replace $A, B, C, D, \mu$ and $\nu$ by $A_{i}, B_{i}, C_{i}, D_{i}, \mu_{i}$ and $\nu_{i}$ respectively, then they will satisfy the relations that correspond to (2.1) -.(2.5). if

Form the free product of $G_{i}$ and $G_{i+1}$ amalgamating $B_{i} \subseteq G_{i}$ with $A_{i+1}$ 트 $G_{i+1}$ as follows :

$$
\begin{array}{rlrl}
b_{i} & =b \gamma_{i}, \quad b \in B, \\
a_{i+1} & =a \gamma_{i+1}, & a \in A,
\end{array}
$$

and if $a \mu=b$ then we put $b_{i}=a_{i+1}$. That is to say, the isomorphism underlying the amalgamation is $\gamma_{i}^{-1} \mu^{-1} \gamma_{i+1} . \quad \gamma_{i}^{-1} \mu^{-1} \gamma_{i+1}$ is defined on $B_{i}$, moreover it is the identical mapping on $B_{i}$. Call this free product

$$
P_{i i+1}=\left\{G_{i} * G_{i+1} ; \quad B_{i}=A_{i+1}\right\}
$$


We then form the free product of $P_{i i+1}$ and $G_{i+2}$ amalgamating $B_{i+1}$ 드 $P_{i i+1}$ with $A_{i+2}$ 드 $G_{i+2}$ according to the isomorphism $\gamma_{i+1}^{-1} \mu^{-1} \gamma_{i+2}$. Call the product

$$
P_{i i+2}=\left\{P_{i i+1} * G_{i+2} ; \quad B_{i+1}=A_{i+2}\right\} \text {. }
$$

More generally we define $P_{i j}$ inductively for any $i<j$ in the following way :

$$
\begin{aligned}
& P_{i i+1}=\left\{G_{i} * G_{i+1} ; \quad B_{i}=A_{i+1}\right\}, \\
& P_{i j}=\left\{P_{i j-1} * G_{j} ; B_{j-1}=A_{j}\right\},
\end{aligned}
$$

where $B_{j-1}$ is amalgamated with $A_{j}$ according to the isomorphism $\gamma_{j-1}^{-1} \mu^{-1} \gamma_{j}$.

We note that we have :

$$
P_{i j} \subseteq P_{h k}
$$

for any $h \leqslant i<j \leqslant k$.

Also by this method of construction $P_{i i+r}$ is isomorphic to $P_{h h+r}$, in an obvious way, for any $i, h$ and positive $r$. This fact will be used later in Lemma 8 .

Now we prove that in $P_{i j}$ the following lemmas are true:

Lemma 5 :

In $P_{i i+1}$ the following relations hold :

$$
\begin{aligned}
& C_{i} \cap B_{i}=C_{i+1} \cap A_{i+1}, \\
& D_{i} \cap B_{i}=D_{i+1} \cap A_{i+1} .
\end{aligned}
$$

Proof.

Let

then

$$
\begin{aligned}
& a_{i} \in C_{i} \cap B_{i}, \\
& a_{i} \gamma_{i}^{-1} \in C \cap B .
\end{aligned}
$$

Since $a_{i} \in B_{i}=A_{i+1}$, that is to say $a_{i}$ is an amalgamated element,

then

$$
a_{i} \gamma_{i}^{-1} \mu^{-1} \gamma_{i+1}=a_{i}
$$

or

$$
a_{i} \gamma_{i}^{-1}=a_{i} \gamma_{i+1}^{-1} \mu
$$

thus

$$
a_{i} \gamma_{i+1}^{-1} \mu \epsilon C \cap B=(C \cap A) \mu \quad \text { by }(2.1) \text {. }
$$

Since $\mu$ is an isomorphism, then

$$
\begin{gathered}
a_{i} \gamma_{i+1}^{-1} \epsilon C \cap A, \\
a_{i} \epsilon(C \cap A) \gamma_{i+1}=C_{i+1} \cap A_{i+1}, \\
C_{i} \cap B_{i} \text { 드 } C_{i+1} \cap A_{i+1} \ldots \ldots
\end{gathered}
$$

If, on the other hand,

$$
a_{i+1} \in C_{i+1} \cap A_{i+1}
$$

then

$$
a_{i+1} \gamma_{i+1}^{-1} \epsilon C \cap A \text {, }
$$

$$
a_{i+1} \gamma_{i+1}^{-1} \mu \epsilon(C \cap A) \mu=C \cap B \text {, by (2.1). }
$$

Since $a_{i+1} \in A_{i+1}=B_{i}$, that is to say $a_{i+1}$ is an amalgamated element, then :

or

$$
a_{i} \gamma_{i}^{-1} \mu^{-1} \gamma_{i+1}=a_{i}
$$

$$
a_{i}^{\prime} \epsilon(C \cap B) \gamma_{i}=C_{i} \cap B_{i},
$$

thus

$$
C_{i+1} \cap A_{i+1} \subseteq C_{i} \cap B_{i}
$$

(2.9) and (2.10) together give

$$
C_{i} \cap B_{i}=C_{i+1} \cap A_{i+1},
$$

which proves (2.8). Similarly one proves (2.9), and the lemma follows. 
Lemma 5 and relation (2.6) give the following :

\section{Corollary:}

In $P_{i j}$ the following relations hold :

$$
\begin{aligned}
& C_{t} \cap B_{t}=C_{t+1} \cap A_{t+1}, \\
& D_{t} \cap B_{t}=D_{t+1} \cap A_{t+1},
\end{aligned}
$$

for all $t=i, i+1, \ldots, j-1$.

\section{Lemma 6.}

If $h \leqslant i<j \leqslant k$ and if we denote by $Q_{i j}$ the subgroup of $P_{h k}$ generated by $C_{i}, C_{i+1}, \ldots, C_{j}-Q_{i i}$, in particular, is $C_{i}-$, and if we similarly denote by $R_{i j}$ the subgroup of $P_{h k}$ generated by $D_{i}, D_{i+1}, \ldots, D_{j}-R_{i i}$, in particular, is $D_{i}-$, then

Proof.

$$
\begin{array}{ll}
Q_{i j}=\left\{Q_{i j-1} * C_{j} ;\right. & \left.C_{j-1} \cap B_{j-1}=C_{j} \cap A_{j}\right\}, \\
R_{i j}=\left\{R_{i j-1} * D_{j} ;\right. & \left.D_{j-1} \cap B_{j-1}=D_{j} \cap A_{j}\right\} .
\end{array}
$$

It is sufficient to prove the lemma for $Q_{i j}$, the proof for $R_{i j}$ will be on similar lines.

Now we shall prove by induction that

$$
\begin{aligned}
& Q_{i j-1} \cap B_{j-1}=C_{j} \cap A_{j}, \\
& Q_{i j}=\left\{Q_{i j-1} * C_{j} ; C_{j-1} \cap B_{j-1}=C_{j} \cap A_{j}\right\},
\end{aligned},
$$

for any $i<j$. The induction is on $j$.

For $j=i+1$ we have

$$
\begin{aligned}
Q_{i i} \cap B_{i} & =C_{i \cap} B_{i} \\
& =C_{i+1} \cap A_{i+1} \quad \text { by Lemma } 5 .
\end{aligned}
$$

Thus by Lemma $1, C_{i}$ and $C_{i+1}$ generate in $P_{i i+1}$ their free product with the amalgamated subgroup $C_{i} \cap B_{i}$ :

$$
Q_{i i+1}=\left\{C_{i} * C_{i+1} ; \quad C_{i \cap} B_{i}=C_{i+1} \cap A_{i+1}\right\}
$$

Assume that (2.13) is true for $j$.

Applying Lemma 2 with $P_{i j}, P_{i j-1}, G_{j}, B_{j-1}, Q_{i j}, Q_{i j-1}, C_{j}, C_{j-1} \cap B_{j-1}$ in the place of $P, G_{1}$, $G_{2}, H, Q, A_{1} A_{2}, B$ respectively we get, because of the first inductive relation of (2.13):

$$
Q_{i} \cap G_{j}=C_{j} \text {. }
$$

Intersecting both sides with $B_{j}$ we get

$$
\begin{aligned}
Q_{i j} \cap B_{j} & =C_{j \curvearrowleft} B_{j} \\
& =C_{j+1} \cap A_{j+1} \quad \text { by (2.11). }
\end{aligned}
$$

Because of this equation, we can apply Lemma 1, with

in the place of

$$
P_{i j+1}, \quad P_{i j}, \quad G_{j+1}, \quad B_{j}, \quad Q_{i j}, \quad C_{j+1}
$$

$$
P, \quad G_{1}, \quad G_{2}, \quad H, \quad A_{1}, A_{2},
$$

and thus $Q_{i j}$ and $C_{j+1}$ generate in $P_{i j+1}$ their free product with the amalgamated subgroup $C_{j} \cap B_{j}=C_{j+1} \cap A_{j+1}$. This completes the proof of Lemma 6 .

\section{Lemma 7.}

There exists an isomorphic mapping $\nu_{i j}$, say, of $Q_{i j}$ onto $R_{i j}$ such that $\nu_{i j}$ extends $\nu_{i}, \nu_{i+1}$, $\ldots, \nu_{j}$. 


\section{Proof.}

The proof is by induction on $j$. We first show that in $Q_{i i+1}$ the map by $\nu_{i}$ of an element in $C_{i} \cap C_{i+1}$ is equal to its map by $\nu_{i+1}$. By the construction of $P_{i i+1}$ we have

$$
G_{i} \cap G_{i+1}=B_{i}
$$

intersecting. both sides with $C_{i} \cap C_{i+1}$ we get

$$
\begin{aligned}
C_{i} \cap C_{i+1} & =B_{i \curvearrowleft} C_{i} C_{i+1} \\
& =A_{i+1} \curvearrowleft C_{i+1} \\
& =B_{i \curvearrowleft} C_{i} \quad \text { by }(2.7) .
\end{aligned}
$$

Let

$$
c_{i} \in C_{i} \cap C_{i+1}=A_{i+1} \cap C_{i+1}=B_{i} \cap C_{i}
$$

then

$$
\begin{aligned}
c_{i} \nu_{i} & =c_{i} \gamma_{i}^{-1} \nu \gamma_{i} \in B_{i} \curvearrowleft D_{i}, \ldots \ldots . . \\
c_{i} \nu_{i+1} & =c_{i} \gamma_{i+1}^{-1} \nu \gamma_{i+1} \in A_{i+1} \cap D_{i+1} .
\end{aligned}
$$

Since $c_{i}$ is an amalgamated element, then

or

$$
\begin{aligned}
c_{i} \gamma_{i}^{-1} \mu^{-1} \gamma_{i+1} & =c_{i} \\
c_{i} \gamma_{i}^{-1} & =c_{i} \gamma_{i+1}^{-1} \mu .
\end{aligned}
$$

$c_{i} \gamma_{i}^{-1} \in B \cap C$, thus applying $\nu$ to the last equation we get

$$
\begin{aligned}
c_{i} \gamma_{i}^{-1} \nu & =c_{i} \gamma_{i+1}^{-1} \mu \nu \\
& =c_{i} \gamma_{i+1}^{-1} \nu \mu \quad \text { by (2.5). }
\end{aligned}
$$

But (2.14) and (2.15) give

$$
\begin{gathered}
c_{i} \gamma_{i}^{-1} \nu=c_{i} \nu_{i} \gamma_{i}^{-1}, \\
c_{i} \gamma_{i+1}^{-1} \nu=c_{i} v_{i+1} \gamma_{i+1}^{-1} .
\end{gathered}
$$

Substituting in (2.16) we get

$$
c_{i} \nu_{i} \gamma_{i}^{-1}=c_{i} \nu_{i+1} \gamma_{i+1}^{-1} \mu_{,}
$$

or $\quad\left(c_{i} \nu_{i}\right) \gamma_{i}^{-1} \mu^{-1} \gamma_{i+1}=\left(c_{i} \nu_{i+1}\right)$.

Thus, according to the rule of amalgamation, we get

This together with the fact that

$$
c_{i} \nu_{i}=c_{i} \nu_{i+1} \text {. }
$$

$$
\left(C_{i} \cap B_{i}\right) \nu_{i}=D_{i} \cap B_{i},
$$

enables us to apply Lemma 4, thus proving the existence of an isomorphic mapping $\nu_{i i+1}$, say, of $Q_{i i+1}$ onto $R_{i i+1}$ such that $\nu_{i i+1}$ extends $\nu_{i}$ and $\nu_{i+1}$.

Suppose that $\nu_{i j}$ maps $Q_{i j}$ isomorphically onto $R_{i j}$ such that $\nu_{i j}$ extends $v_{i}, v_{i+1}, \ldots, \nu_{i}$. For any element

$$
c \in Q_{i j} \frown C_{j+1},
$$

we get $c \in C_{j} \cap B_{j}=C_{j+1} \cap A_{j+1}$, since by Lemma 6 we have

$$
Q_{i j} \cap C_{i+1}=C_{j} \cap B_{j}=C_{j+1} \cap A_{j+1} .
$$

For such an element $c$, we proved above that

$$
c \nu_{j}:=c \nu_{j+1} .
$$


Since $\nu_{i j}$ extends $\nu_{j}$, that is to say, since

then

$$
\begin{aligned}
c \nu_{j} & =c \nu_{i j}, \\
c \nu_{i j} & =c \nu_{j+1} .
\end{aligned}
$$

Thus it is legitimate to apply Lemma 4 which proves the existence of an isomorphic mapping $v_{i j+1}$, say, of

$$
Q_{i j+1}=\left\{Q_{i j} * C_{j+1} ; \quad C_{j} \cap B_{j}=C_{j+1} \cap A_{j+1}\right\}
$$

onto $\quad R_{i j+1}=\left\{R_{i j} * D_{j+1} ; D_{j} \cap B_{j}=D_{j+1} \cap A_{j+1}\right\}$,

which extends $\nu_{i j}$ and $v_{j+1}$ and thus extends $v_{i}, \nu_{i+1}, \ldots, \nu_{j+1}$. This completes the proof of the lemma.

Now we form

$$
P^{*}=\bigcup_{n=1}^{\infty} P_{-n,+n}
$$

Define the mapping $\mu^{*}$ as follows : For any $g_{i} \in G_{i}$, if $g_{i} \gamma_{i}^{-1}=g$ and $g \gamma_{i+1}=g_{i+1}$, then we put

in other words, on $G_{i}$,

$$
g_{i} \mu^{*}=g_{i+1}
$$

$$
g_{i} \mu^{*}=g_{i} \gamma_{i}^{-1} \gamma_{i+1}
$$

We prove then the following

\section{Lemma 8.}

The mapping $\mu^{*}$ generates an automorphism of $P^{*}$ which extends every $\mu_{i}$. Proof.

That $\mu^{*}$ generates a mapping of $P^{*}$ onto itself is obvious. It is also consistent for if

and if

$$
g_{i} \in G_{i} \cap G_{i+1}=B_{i}=A_{i+1},
$$

$$
\begin{aligned}
& g_{i} \gamma_{i}^{-1}=g, \\
& g \gamma_{i+1}=g_{i+1},
\end{aligned}
$$

then

$$
g_{i} \mu^{*}=g_{i+1} \in B_{i+1} \text {. }
$$

If, on the other hand,

$$
\begin{aligned}
& g_{i} \gamma_{i+1}^{-1}=g^{\prime}, \\
& g^{\prime} \gamma_{i+2}=g_{i+2},
\end{aligned}
$$

then

$$
g_{i} \mu^{*}=g_{i+2} \in A_{i+2}
$$

Since $g_{i}$ is an amalgamated element, then

But

$$
g_{i} \gamma_{i}^{-1} \mu^{-1} \gamma_{i+1}=g_{i} \text {. }
$$

and

$$
\begin{gathered}
g_{i} \gamma_{i}^{-1}=g=g_{i+1} \gamma_{i+1}^{-1}, \\
g_{i} \gamma_{i+1}^{-1}=g^{\prime}=g_{i+2} \gamma_{i+2}^{-1} .
\end{gathered}
$$

These last three equations together give

$$
g_{i+1} \gamma_{i+1}^{-1} \mu^{-1} \gamma_{i+2}=g_{i+2}
$$

Thus $g_{i+1}$ is to be amalgamated with $g_{i+2}$. This proves the consistency of $\mu^{*}$. 
That $\mu^{*}$ generates an automorphism of $P^{*}$ follows from the fact that $P_{i i+1}$ is isomorphic to $P_{i+1 i+2}$ under the mapping generated by $\gamma_{i}^{-1} \gamma_{i+1}$ and $\gamma_{i+1}^{-1} \gamma_{i+2}$.

To prove that $\mu^{*}$ extends every $\mu_{i}$, we take an arbitrary element $a_{i} \in A_{i}$, then by the definition of $\mu^{*}$ and $\mu_{i}$ we get

$$
\begin{gathered}
a_{i} \mu^{*}=a_{i} \gamma_{i}^{-1} \gamma_{i+1} \in A_{i+1}, \\
a_{i} \mu_{i}=a_{i} \gamma_{i}^{-1} \mu \gamma_{i} \in B_{i} .
\end{gathered}
$$

Since

$$
\begin{aligned}
\left(a_{i} \gamma_{i}^{-1} \mu \gamma_{i}\right) \gamma_{i}^{-1} \mu^{-1} \gamma_{i+1} & =a_{i} \gamma_{i}^{-1} \gamma_{i+1}, \\
\left(a_{i} \mu_{i}\right) \gamma_{i}^{-1} \mu^{-1} \gamma_{i+1} & =\left(a_{i} \mu^{*}\right)
\end{aligned}
$$

then by the rule of amalgamation we get

$$
a_{i} \mu_{i}=a_{i} \mu^{*}
$$

This completes the proof of the lemma.

The group $P^{*}$ contains the subgroups

$$
\begin{aligned}
& C^{*}=\left\{\ldots, C_{-1}, C_{0}, C_{1}, \ldots\right\}, \\
& D^{*}=\left\{\ldots, D_{-1}, D_{0}, D_{1}, \ldots\right\} .
\end{aligned}
$$

It is clear that $\mu^{*}$ maps $C^{*}$ onto itself and maps $D^{*}$ onto itself.

Define the mapping $\bar{\nu}$ of $C^{*}$ onto $D^{*}$ as follows : for $c \epsilon C^{*}$, that is to say $c \epsilon Q_{i j}$ for some suitable $i$ and $j$, put

$$
c \bar{\nu}=c \nu_{i j} .
$$

$\bar{\nu}$ is thus an isomorphism of $C^{*}$ onto $D^{*}$, which extends the $\nu_{i}$ since the $\nu_{i j}$ does.

It is clear that for any $c^{*} \epsilon C^{*}, c^{*} \bar{\nu} \mu^{*}$ is defined, and since $c^{*} \mu^{*} \epsilon C^{*}$ then $c^{*} \mu^{*} \bar{\nu}$ is also defined. Moreover, we have :

Lemma 9.

for any $c^{*} \epsilon C^{*}$.

$$
c^{*} \bar{\nu} \mu^{*}=c^{*} \mu^{*} \bar{\nu}
$$

Proof.

Let

Since $\bar{\nu}$ extends all the $\nu_{i}$ then

$$
c^{*}=\prod_{i} c_{i}, \quad c_{i} \in C_{i}
$$

On the other hand,

$$
\begin{aligned}
c^{*} \bar{\nu} & =\prod_{i} c_{i} \nu_{i} \\
& =\prod_{i} c_{i} \gamma_{i}^{-1} \nu \gamma_{i}, \\
c^{*} \bar{\nu} \mu^{*} & =\prod_{i} c_{i} \gamma_{i}^{-1} \nu \gamma_{i} \gamma_{i}^{-1} \gamma_{i+1} \\
& =\prod_{i} c_{i} \gamma_{i}^{-1} \nu \gamma_{i+1} .
\end{aligned}
$$

$$
\begin{aligned}
c^{*} \mu^{*} \bar{\nu} & =\prod_{i} c_{i} \gamma_{i}^{-1} \gamma_{i+1} \nu_{i+1} \\
& =\prod_{i} c_{i} \gamma_{i}^{-1} \gamma_{i+1} \gamma_{i+1}^{-1} \nu \gamma_{i+1} \\
& =\prod_{i} c_{i} \gamma_{i}^{-1} \nu \gamma_{i+1}
\end{aligned}
$$

Thus $c^{*} \bar{\nu} \mu^{*}=c^{*} \mu^{*} \bar{\nu}$, and the lemma follows. 
Now form the group generated by $P^{*}$ and an element $s$, call this group

$$
\widehat{P}=\left\{P^{*}, s\right\} \text {, }
$$

and define

$$
s^{-1} p^{*} s=p^{*} \mu^{*} \quad \text { for all } p^{*} \in P^{*} ;
$$

$s$ thus induces an inner automorphism of $\widehat{P}$.

Equation (2.17) then translates into

$$
s^{-1}\left(c^{*} \bar{\nu}\right) s=\left(s^{-1} c^{*} s\right) \bar{\nu},
$$

that is, the inner automorphism $s$ commutes with the partial automorphism $\bar{\nu}$.

If we, moreover, define

then $\bar{\nu}$ becomes an isomorphism of

$$
s \bar{\nu}=s,
$$

onto

$$
\begin{aligned}
& \widehat{C}=\left\{C^{*}, s\right\} \\
& \widehat{D}=\left\{D^{*}, s\right\},
\end{aligned}
$$

and it also commutes with $s$.

At this stage we mention a theorem proved by G. Higman, B. H. Neumann and $H$. Neumann (2, Theorem 1), namely, the following

\section{Theorem 2.}

Let $\mu$ be an isomorphism of a $\operatorname{subgroup} A$ of a group $G$ onto a second $\operatorname{subgroup} B$ of $G$. Then there exists a group $H$ containing $G$, and an element $t$ of $H$, such that the transform by $t$ of any element of $A$ is its image under $\mu$ :

$$
t^{-1} a t=a \mu \text { for all } a \epsilon A \text {. }
$$

Applying this theorem with $\bar{\nu}, \widehat{C}, \widehat{D}, \widehat{P}$ taking the place of $\mu, A, B, G$ respectively, we can embed $P$ in a group

such that

$$
G^{*}=\{\widehat{P}, t\}
$$

$$
t^{-1} \hat{c} t=\hat{c} \text { for any } \hat{c} \in \widehat{C},
$$

that is to say $t$ induces an inner automorphism of $G$ which extends $\bar{v}$ and thus extends $v$.

Putting $c=s$ in (2.18) we get

$$
\begin{aligned}
t^{-1} s t & =s \bar{\nu} \\
& =s \quad \text { by definition. }
\end{aligned}
$$

Thus $s$ and $t$ commute and $G^{*}$ has the required properties. This completes the proof of Theorem 1.

Remark. We note that conditions (2.1)-(2.4) of Theorem 1 are not necessary; for if we take the subgroups $A, B, C, D$ of $G$ such that

$$
A=C \neq\{1\}, \quad B=D, \quad A \cap B=\{1\},
$$

and take $\nu$ to be the same mapping as $\mu$, then the conclusion of the theorem is known to be valid (by Theorem 2$)$, although $(A \cap D) \mu \neq B \cap D$.

A special case of Theorem 1 is the following:

Corollary :

Sufficient for $\mu$ and $\nu$ to be extendable to two commutative inner automorphisms of one and the same group is that

$$
A \cap C=B \cap C=A \cap D=B \cap D=\{1\} .
$$

For then equations (2.1)-(2.5) will be trivially satisfied. 


\section{REFERENCES}

(1) Baer, Reinhold, "Free sums of groups and their generalizations. An analysis of the associative law," Amer. J. Math., 71, (1949), 706-742.

(2) Higman, G., Neumann, B. H., and Neumann, H., " Embedding theorems for groups," J. London Math. Soc., 24 (1949), 247-254.

(3) Neumann, Hanna, "Generalized free products with amalgamated subgroups," Amer. J. Math., 70, (1948), 590-625.

Department of Mathematics

The UnIVersity

MafChester

and

Faculty of Science

The UNTVERSTTY

AlexandRIA 